\title{
Perspectives d'utilisation de la génétique pour la sélection des Penicillium
}

\author{
par \\ Joseph P. GUIRAUD* et Pierre GALZY \\ Laboratoire de Recherches de la Chaire de Génétique \\ Ecole Nationale Supérieure Agronomique \\ Centre de Recherches Agronomiques de Montpellier \\ Institut National de la Recherche Agronomique \\ (34) Montpellier (France)
}

Certaines espèces de Penicillium ont été très utilisées en génétique fondamentale : Penicillium chrysogenum Thom, Penicillium notatum Westling, Penicillium expansum Link et Penicillium italicum Wehmer [4]. Ces espèces ne donnent pas un cycle sexuel complet mais de nombreux auteurs [3-20-23-24-33] ont montré qu'elles possèdent un cycle parasexuel semblable à celui découvert en $1952 \mathrm{chez}$ Aspergillus nidulans (Eidam) Winter par Pontecorvo et Roper [22]. Une des applications importantes du cycle parasexuel est l'amélioration génétique des caractéristiques industrielles (production de pénicilline, etc.) de Penicillium chysogenum et de Penicillium notatum [11-12-13-14].

En fromagerie, on utilise fréquemment des Penicillium : Penicillium roqueforti Thom, Penicillium casei Staub, Penicillium camenberti Thom et Penicillium caseicola Bainer [31]. La présence du cycle parasexuel n'a pas encore été mise en évidence dans les souches industrielles appartenant à ces espèces. Il est cependant probable que ce cycle existe car la parenté est grande entre les Penicillium roqueforti et les Penicillium notatum (ce sont tous deux des assymétrica de la sous-vection vélutina). On peut supposer que la parasexualité fonctionne dans les deux cas.

L'utilisation du cycle parasexuel pour la sélection industrielle pourrait revêtir dans l'avenir une grande importance. Nous en avons résumé dans le schéma $\mathrm{n}^{\circ} 1$ les différents stades dont les principaux sont d'après Pontecorvo [19] :

- Formation d'un hétérocaryon.

- Formation d'un diplö̈de.

\footnotetext{
* Service de Microbiologie (Sciences et Techniques de l'Alimentation), Université des Sciences et Techniques du Languedoc (34) Montpellier (France).
} 


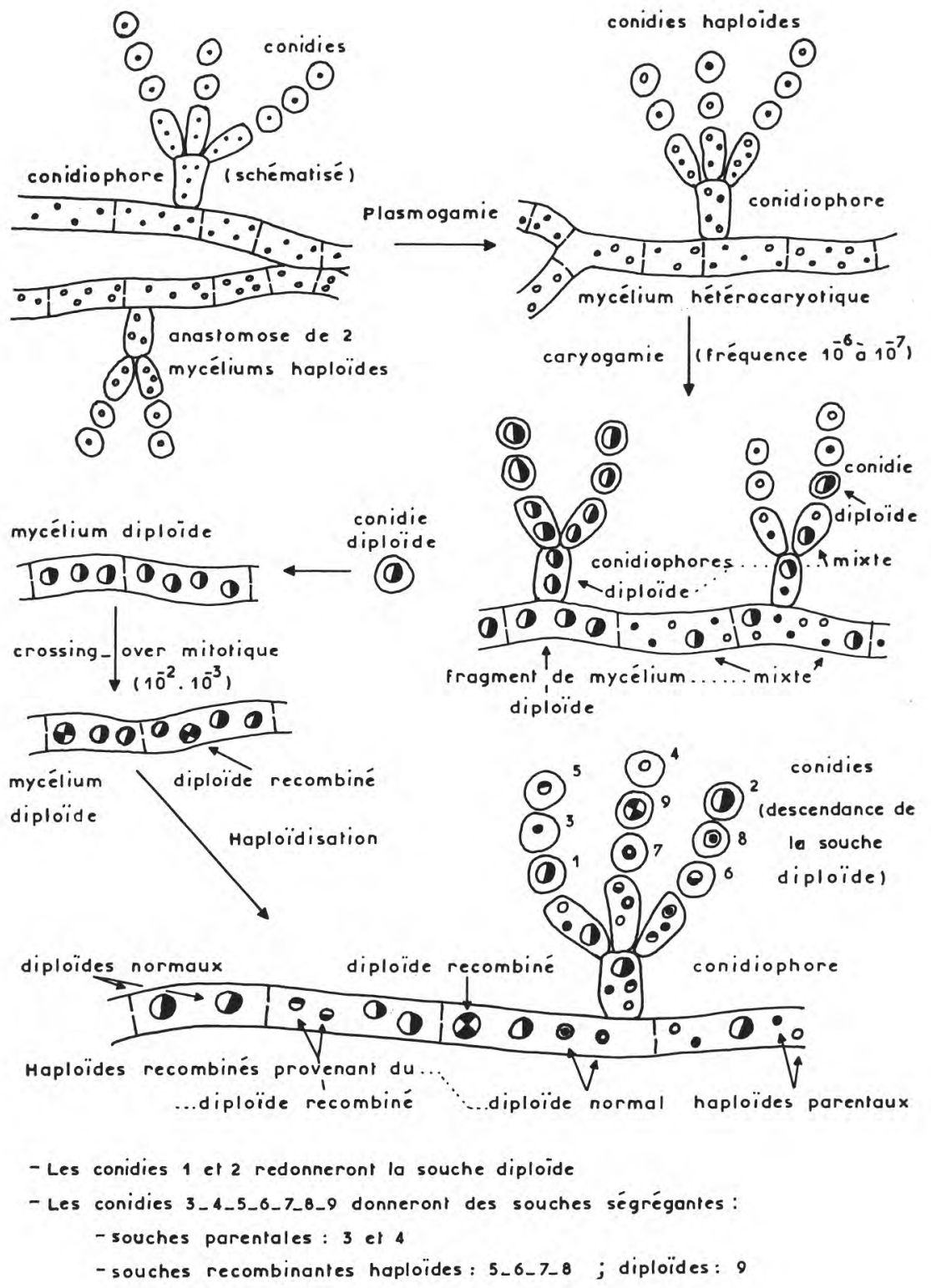

Schéma 1

Les différentes étapes du cycle parasexuel chez les Penicillium. 
- Crossing over mitotique.

- Haploïdisation.

Le but de ce travail est la description des différentes étapes du cycle, dont nous rappellerons les principales techniques d'étude.

\section{I. - ETAPE PRELIMINAIRE : MARQUAGE GENETIQUE DES SOUCHES}

Pour suivre et étudier les diverses étapes du cycle parasexuel, il est indispensable d'avoir des souches dont les chromosomes sont marqués grâce à des gênes responsables d'un phénotype facilement repérable. Des mutants peuvent être obtenus en traitant les conidies du Penicillium par un agent mutagène physique ou chimique [1-17-25]. Les mutations mises en évidence peuvent être morphologiques : couleur des conidies, nanisme des colonies, etc. [24]. Elles peuvent porter également sur la résistance à des inhibiteurs de croissance et il suffit pour les cribler, d'étaler un grand nombre de conidies à la surface d'un milieu de croissance contenant une dose toxique de l'inhibiteur ( $10^{3}$ conidies par boîte). Les mutants auxotrophes sont plus difficiles à mettre en évidence. Ils sont sélectionnés en cultivant à la surface d'un milieu minimum les conidies traitées. Les prototrophes sont seuls capables de germer. On ajoute ensuite à la surface de la boîte une couche de milieu complet contenant une dose toxique de nystatine. Cet antibiotique tue les mycéliums en croissance active mais n'a pas d'action sur les conidies auxotrophes incapables de pousser. Après 3 semaines d'incubation à $25^{\circ} \mathrm{C}$, la nystatine perd son activité. Les auxotrophes peuvent alors germer et donner des colonies faciles à prélever [8-17].

\section{II. - FORMATION ET CRIBLAGE DES HETEROCARYONS}

Deux mycéliums haploïdes peuvent s'anastomoser ; il se produit un échange de noyaux. Si les deux mycéliums ont des génotypes différents, il se forme un mycélium hétérocaryotique.

Le criblage des hétérocaryons s'effectue de façon classique par sélection de prototrophes [24]. Deux souches auxotrophes différant par leurs besoins nutritifs sont cultivées en mélange dans un milieu liquide complet. Les mycéliums obtenus sont repiqués par isolement de simples hyphes à la surface d'un milieu gélosé minimum. Chaque fragment de mycélium hétérocaryotique donnera naissance à une colonie. En effet, par le jeu de la dominance, les noyaux en présence contiennent toute l'information génétique nécessaire pour rendre ce mycélium prototrophe.

Une autre technique est parfois utilisée lorsque les deux souches à croiser présentent une croissance faible sur milieu riche (souches 
naines). Elles sont alors ensemencées sur une même boîte de Pétri contenant un milieu complet. Au point de jonction des deux mycéliums, il se forme des hétérocaryons, facilement repérables par la vigueur de leur développement.

Le mycélium hétérocaryotique est repiqué et conservé sur milieu minimum. Il convient de surveiller que ses caractéristiques morphologiques restent constantes. Il est important de le repiquer uniquement à partir de simples hyphes ; les conidies émises par ce mycélium sont uninuclées et si on les étale sur milieu riche on obtient un retour aux deux types parentaux. Ces résultats permettent de contrôler le caractère hétérocaryotique d'un mycélium.

\section{III. - FORMATION ET CRIBLAGE DES DIPLOIDES}

Des fusions de noyaux peuvent se produire au sein du mycélium hétérocaryotique en donnant des noyaux diploïdes hétérozygotes. Cet événement est rare (fréquence $10^{-6}$ à $10^{-7}$ ). Parfois, les noyaux haploïdes et diploïdes continuent à se multiplier dans le même mycélium. Parfois aussi, il se produit une ségrégation : il apparaît des secteurs coloniaux où le mycélium est diploïde hétérozygote et des secteurs où le mycélium est haploïde hétérocaryotique. Il est donc possible à partir d'un mycélium hétérocaryotique d'obtenir des conidies haploïdes (cas général) ou diploïdes (après caryogamie).

Si les deux souches parentales sont auxotrophes, les conidies haploïdes sont également auxotrophes, alors que les diploïdes sont prototrophes comme le mycélium hétérocaryotique.

Pour cribler les diploïdes, une suspension de conidies issues de ce mycélium est étalée à la surface d'un milieu minimum : seule les conidies diploïdes donneront des colonies.

Dans le cas de deux souches parentales présentant une différence de pigmentogénèse on peut obtenir des conidies de la couleur du type sauvage (vertes) qui sont diploïdes, alors que les conidies haploïdes sont du type muté (par exemple jaunes et blanches). Une colonie verte est prélevée et purifiée par micro-manipulation.

Les techniques utilisées sont dues à Roper [27].

D'autre part, il a été vérifié que les conidies diploïdes sont en général plus grosses que les conidies haploïdes [15]. Ce critère, bien que n'étant pas absolu, est utilisé aussi pour le criblage des diploïdes : on prélève par micro-manipulation quelques grosses conidies que l'on conserve par repiquage sur milieu complet.

\section{IV. - DESCENDANCE DU DIPLOIDE ET CRIBLAGE DES RECOMBINANTS}

Dans la descendance de l'hétérozygote diploïde, il apparaît des colonies dans lesquelles les caractères récessifs s'expriment. Ces 


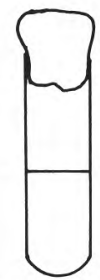

suspension de conidies diploïdes.

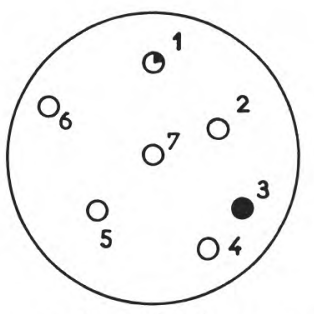

Etalement sur milieu gélosé complel

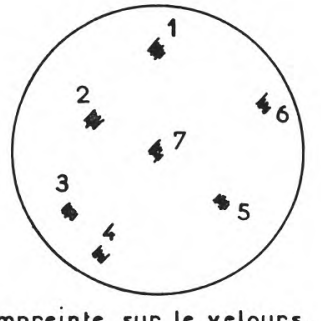

- Isolement direct de la colonie 3 el du secteur variant morphologique de la $\mathrm{co}$.

lonie 1.

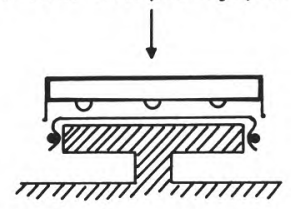

Réplica_plating ( 1 ère phase)

Empreinte sur le velours.

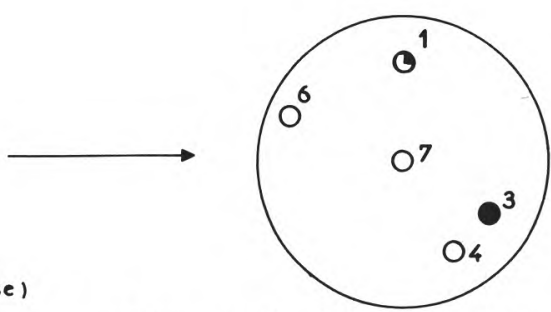

Réplica_plating ( 2 ème phase)

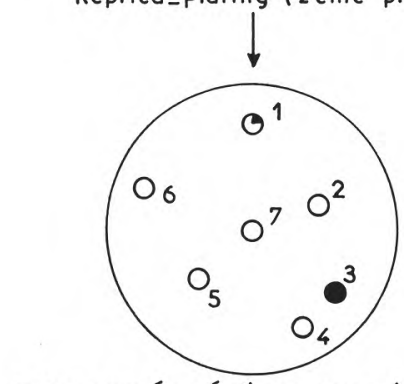

Culture aprés réplique sur milieu minimum

- Les colonies 2 el 5 ne donnent pas de

croissance sur milieu minimum et sont donc auxotrophes.

- Isolement de la colonie 2 er de la colonie 5 aprés obtention du résultat sur milieu minimun.

Culture aprés réplique sur milieu complel gélosé.

\section{Schéma 2}

Criblage des ségrégants issus de l'hétérozygote diploïde. 
ségrégants peuvent être soit des haploïdes de type parental, soit des recombinants, dont l'isolement est essentiel du point de vue industriel.

Les recombinants peuvent être de divers types [18] :

- soit des haploïdes, issus d'une recombinaison interchromosomique ;

- soit des diploïdes ayant subi un crossing over mitotique, c'està-dire une recombinaison intrachromosomique ;

- soit des haploïdes provenant de ces diploïdes recombinés.

Il convient de citer également la possibilité de trouver parmi les ségrégants des diploïdes devenus homozygotes pour une paire de chromosomes homologues, les autres paires restant hétérozygotes. Ils apparaissent par l'entremise d'un mécanisme appelé « mitotic non disjonction » [21-29].

Le criblage des ségrégants peut s'effectuer en étalant sur un milieu complet des conidies diploïdes (schéma $n^{\circ} 2$ ). Il apparaît, parmi les colonies normales, des colonies exprimant un caractère morphologique récessif, et d'autres présentant seulement des secteurs de ce type. La méthode de " replica plating " permet de rechercher les ségrégants auxotrophes comme l'indique aussi le schéma no 2 [24].

Une autre méthode pour cribler les ségrégants consiste à étaler sur un milieu complet, les conidies du diploïde et de tester systématiquement chacune des colonies obtenues au point de vue morphologique, exigences nutritionnelles, etc. à l'aide des techniques précitées. Dans cette éventualité le travail est naturellement beaucoup plus long mais a l'avantage de permettre de distinguer les recombinants des haploïdes parentaux.

Les ségrégants ayant été obtenus, il convient de mettre en évidence les recombinants, ainsi que de déterminer dans le cas de ces derniers si on se trouve en présence d'une haploïdisation spontanée (recombinants haploïdes) ou d'un crossing over mitotique (recombinants diploïdes et leur descendance haploïde). Le criblage s'effectue à l'aide des tests mentionnés plus haut et utilise les modalités d'expression des marqueurs récessifs.

Dans le cas d'un crossing over mitotique, la plupart des marqueurs récessifs présents à l'état hétérozygote chez le diploïde, resteront masqués ; seuls les marqueurs portés par le chromosome touché par le crossing over apparaîtront pourvu qu'ils soient sur la portion de chromosome recombiné : en effet le crossing over mitotique consiste en une recombinaison intrachromosomique qui ne touche qu'un seul chromosome [21]. Le crossing over mitotique se produit comme le crossing over méiotique au stade 4 brins [32]. Deux seulement des 4 chromatides homologues en présence sont touchées. La recombinaison mitotique est réciproque [30]. Le schéma $\mathrm{n}^{\circ} 3$ en montre les étapes et les conséquences. Les crossing over se produisent au cours des mitoses avec une fréquence de $10^{-2}$ à $10^{-3}$ par division 


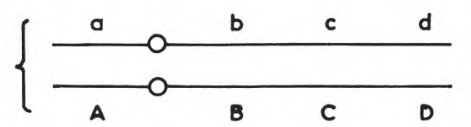

chromosomes

homologues

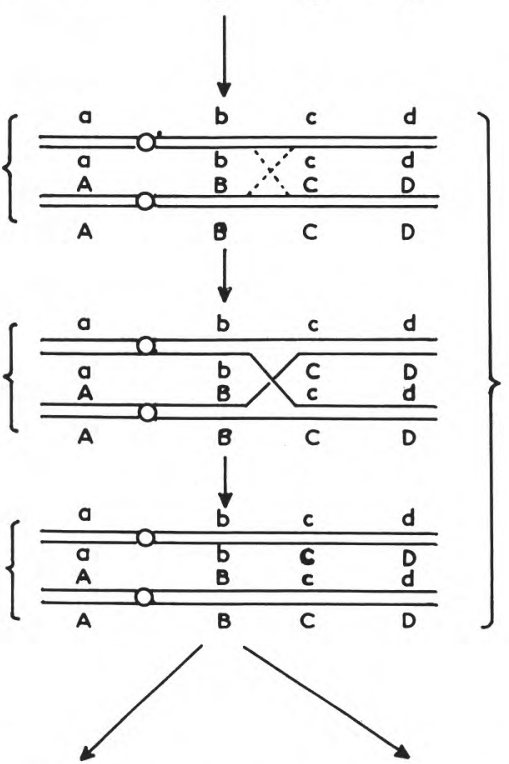

Crossing over

mitorique

(stade 4 brins)

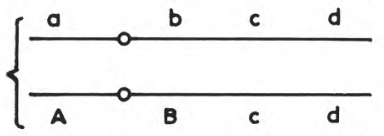

$\left[\begin{array}{c}A B C d \\ \downarrow\end{array}\right.$

ségrégant = recombinant de phénolype
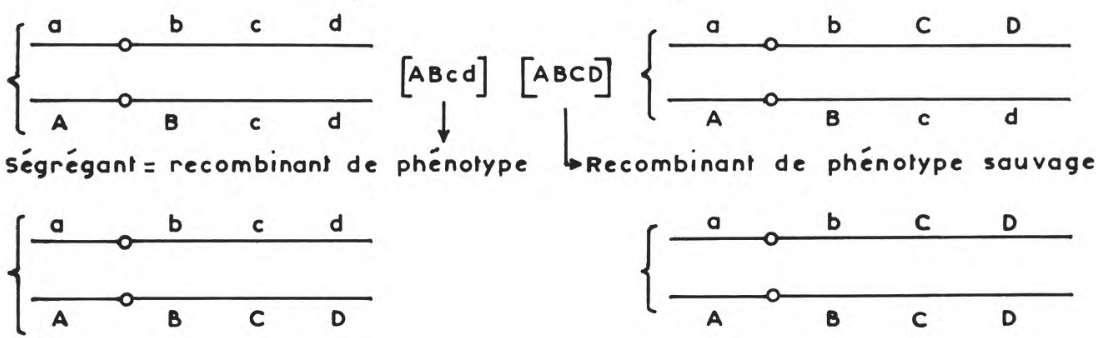

Saurage $[A B C D]$

Recombinant de phénotype sauvage [A B C D ]

Schéma 3

Crossing over mitotique

Sur les 4 types possibles de diploïdes, 3 sont de phénotype sauvage (A B C D) et 1 est un ségrégant de phénotype récessif ( $\mathrm{A} \mathrm{B} \mathrm{c}$ d). Il s'agit bien d'un diploïde recombinant ayant subi un crossing over mitotique car un haploïde spontané aurait pour phénotype $(\mathrm{a} \mathrm{b} \mathrm{cd})$. 
de noyau. En général il n'y a qu'un seul crossing over au cours d'une mitose.

Certaines substances chimiques sont capables d'induire des crossing over mitotiques : les agents alkylants sont particulièrement actifs à cet égard [16]. D'autres agents mutagènes (rayons X, ultra-violets, radiations ionisantes) produisent également un accroissement de la fréquence des crossing over, mais leur action est moins nette [10]. Cette action est indépendante de leur action mutagène.

L'haploïdisation permet au contraire à un grand nombre de marqueurs récessifs d'apparaître : en effet, elle correspond à une recombinaison interchromosomique. La souche haploïde hérite de chromosomes provenant des 2 génomes parentaux [9]. Les caractères contrôlés par des gênes portés par un même chromosome ségrègent simultanément. L'haploïdisation est un événement rare mais spontané (fréquence de $10^{-3}$ par division nucléaire). Il n'y a pas de méïose. Il se produit une perte spontanée de chromosomes qui provoque la transformation du noyau diploïde en noyau haploïde balancé avec éventuellement formation transitoire d'un aneuploïde [6-26]. La dissémination des noyaux haploïdes est réalisée soit par la formation d'un secteur à mycélium haploïde dans une colonie, soit par l'émission de conidies à noyau haploïde. Certaines substances comme la p. fluorophénylalanine favorisent l'haploïdisation [2-7].

L'haploïdisation peut se produire chez des diploïdes qui n'ont pas subi de crossing over mitotique : elle provoque alors uniquement une recombinaison génétique interchromosomique ; il apparaît des haploïdes parentaux, et de nombreux recombinants. Si l'haploïdisation se produit après un crossing over mitotique, le cycle parasexuel est complet; outre la recombinaison interchromosomique il se produit une recombinaison intrachromosomique et il apparaît plusieurs types d'haploïdes (schéma n 4 ).

Malheureusement, les crossing over mitotiques étant peu nombreux, la recombinaison intrachromosomique se produit en général pour un seul chromosome à la fois. L'amélioration génétique est donc plus longue et plus difficile à travers le cycle parasexuel qu'à travers la reproduction sexuée [28].

\section{CONCLUSION}

La sélection d'un micro-organisme industriel a pour but l'obtention d'une souche présentant les caractéristiques permettant la rentabilité maximum. Les critères de sélection sont difficiles à définir et sont toujours très variés : vitesse de croissance, rendement, influence sur la qualité des produits, etc.

Cette sélection implique une variation génétique. Il est possible le plus souvent de faire appel à la variation naturelle. Il existe dans 


\section{Parents haploïdes}

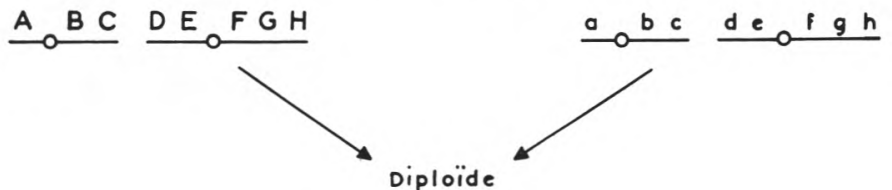

Diploide

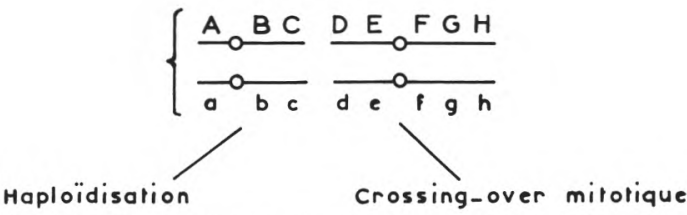

(recombinaison interchromoso- (recombinaison intrachromosomique)

2 haploïdes parentaux

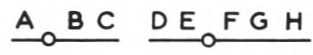

et

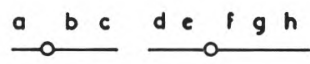

2 haploides

recomb. interchrom.

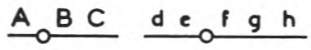

ef

$\underbrace{b}_{-}$c DE $F G H$
4 diploïdes" possibles (schéma 3 ) dont un ségrégant recombinant

$$
\overbrace{a}^{\infty} \frac{B C}{\text { Haploidisation }}
$$

(recombinaison interchromosomique)

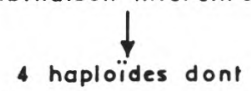

1 parental

3 recombinants

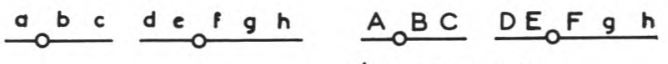

(intrachrom.)

$$
\mathrm{A}_{0}^{\mathrm{B} C} \mathrm{der} \mathrm{g} h
$$

(interchrom.)

$$
\overbrace{\text { (inter el inirachrom.) }}^{a b c} \text { DE }
$$

\section{Schéma 4}

Haploïdisation avec ou sans crossing over mitotique

* Il faut noter que 2 des diploïdes non ségrégants sont recombinants malgré leur phénotype sauvage et peuvent donc donner des haploïdes recombinants intrachromosomiques. 
la nature une grande variété de souches; il est relativement facile de les isoler et de les étudier. Dans le cas d'une industrie ancienne d'origine artisanale il est fréquent que la variété génétique naturelle soit considérable (cas de l'industrie du Roquefort). L'exploitation des types biologiques naturels est toujours une première étape indispensable. Il est cependant possible d'en créer de nouveaux au laboratoire grâce à la mutagénèse. Cette méthodologie permet d'obtenir une variation importante à partir d'une souche unique, ainsi que des caractéristiques rarement rencontrées dans la nature. Des résultats ont été obtenus par cette voie dans le cas de l'industrie des antibiotiques (Pénicilline) [1].

La sélection ainsi définie a cependant des possibilités limitées : on ne peut que choisir entre des types biologiques naturels ou obtenus par mutation. La véritable création n'est possible que par l'entremise de la recombinaison génétique : elle seule permet le regroupement sur une seule souche de caractéristiques intéressantes du point de vue industriel.

Actuellement les utilisations de la génétique pour l'amélioration des souches industrielles sont peu nombreuses. Les seules tentatives se situent dans l'industrie des antibiotiques où les résultats sont prometteurs [5]. Il serait souhaitable d'étudier le cycle parasexuel des Penicillium de l'industrie fromagère, tout en obtenant des souches marquées. Il est en effet permis d'espérer qu'il sera fait appel dans l'avenir à la recombinaison génétique pour leur amélioration.

\section{Ré s u m é}

La recombinaison génétique est possible chez les Penicillium grâce au cycle parasexuel.

Ce cycle a été mis en évidence par Pontecorvo et Roper ; il devrait permettre d'utiliser les techniques génétiques pour l'amélioration des souches industrielles, Cet article bibliographique résume les diverses techniques utilisées pour l'étude du cycle parasexuel.

\section{S u m m a r y}

Genetic recombination in Penicillium is made possible by its parasexual cycle.

This cycle, which has been demonstrated by Pontecorvo and Roper will allow the use of genetic techniques to improve industrial strains. The various techniques used for studying the parasexual cycle are presented in this paper. 


\section{Bibliographie}

[1] Backus (M. P.), Stauffer (J. F.) (1955). - Mycologia, 47, 4, 429.

[2] Ball (C) (1971). - J. Gen. Microbiol., 66, 63.

[3] Barron (G. L.) (1962). - Canad. J. Botany, 40, 1603.

[4] ESSER (K.), KuENEN (R.) (1967). - Dans « Genetic of fungi ». Springer Verlag, New-York Inc.

[5] Holt (G.), Macdonald (K. D.) (1968). - Nature, 219, 636.

[6] KAFER (E.) (1961). - Genetics, 46, 1581.

[7] LhoAs (P.) (1961), - Nature, 190, 734.

[8] Macdonald (K. D.) (1968). - Genet. Res. Camb., 11, 327.

[9] Macdonald (K. D.) (1968). - Mutation Res., 5, 302.

[10] Macdonald (K. D.) (1971). - J. Gen. Microbiol., 67, 247.

[11] Macdonald (K. D.), Hutchinson (J. M.), Gillet (W. A.) (1963), - J. Gen. Microbiol., 33, 365 .

[12] Macdonald (K. D.), Hutchinson (J. M.), Gillet (W. A.) (1963), - J. Gen. Microbiol., 33, 375 .

[13] Macdonald (K. D.), Hutchinson (J. M.), Gillet (W. A.) (1963), - J. Gen. Microbiol., 33, 385.

[14] Macdonald (K. D.), Hutchinson (J. M.), Gillet (W. A.) (1964). - Antonie van Leeuwenhoek, 30 - 2, 209.

[15] Macdonald (K. D.), Hutchinson (J. M.), Gillet (W. A.) (1965). - Genetica, 36, 378.

[16] Morpurgo (G.) (1963). - Genetics, 48, 1259.

[17] Pontecorvo (G.) (1953). - Advanc. Genet., 5, 141.

[18] Pontecorvo (G.) (1954). - Caryologia, vol. suppl.

[19] Pontecorvo (G.) (1956). - Ann. Rev. Microbiol., 1, 393.

[20] Ponteconvo (G.), Gemmel (A. R.) (1944). - Nature, 154, 514.

[21] Ponteconvo (G.), Kafer (E.) (1958). - Advanc. Genet., 9, 71.

[22] Ponteconvo (G.), Roper (J. A.) (1952). - J. Gen. Microbiol., 6, vii.

[23] Ponteconvo (G.), Sermonti (G.) (1953). - Nature, 172, 126.

[24] Pontecorvo (G.), Sermonti (G.) (1954). - J. Gen. Microbiol., 11, 94.

[25] Pontecorvo (G.), Roper (J. A.), Forbes (E.) (1953). - J. Gen. Microbiol, 8, 198.

[26] Pontecorvo (G.), Tan Gloor, Forbes (E.) (1954). - J. Genet., 25, 226.

[27] Roper (J. A.) (1952). - Experientia, 8, 14.

[28] Roper (J. A.) (1964), - Dans " Recent Progress in Microbiology VIII ». University of Toronto press.

[29] Roper (J. A.) (1965). - Dans « Biogenesis of antibiotic substances ». Publishing House of the Czechoslovak Academy of Sciences.

[30] Roper (J. A.), PritchaRd (R. H.) (1955). - Nature, 175, 639.

[31] Sмith (G.) (1969). - Dans " An introduction to industrial mycology ". Edward Arnold Ltd, London.

[32] Stern (C.) (1936). - Genetics, 21, 625.

[33] Str $\varnothing$ manas (Ø.), Beraha (L.) (1964). - Canad. J. Botany, 42, 423. 